\section{Reflexões sobre a anticoncepção na adolescência no Brasil}

\section{Considerations on contraceptive methods used by adolescents in Brazil}

Leila Maria Vieira 1

Sandra de Oliveira Saes 2

Adriana Aparecida Bini Dória 3

Tamara Beres Lederer Goldberg 4

\section{Abstract}

Considerations about the consequences of contraceptives use by adolescents, are made, based on National and international studies warn against early sexual life exposing adolescents to the risks of pregnancy and sexualy transmissible diseases including AIDS. For the most adolescents informations on contraceptives are offered, the pill and the condom being the most popular and used methods. However, a high inadequacy level of contraceptive methods was determined in addition to the lack of guidance and assistance services.

Such a situation alerts to the need to have health and education professionals, family and community involved to warrant adolescents an improved life quality.

Key words Adolescence, Pregnancy, Contraception

\section{Resumo}

Faz-se uma reflexão sobre a contracepção e suas conseqüências na adolescência, baseada em trabalhos nacionais e internacionais alertam para a precocidade do início da atividade sexual, a qual expõe os adolescentes aos riscos da gravidez e das doenças sexualmente transmissíveis e AIDS. Verificase que a maioria dos adolescentes recebe informações sobre contracepção, sendo a pílula e o preservativo os mais conhecidos e utilizados. Porém, registram-se elevada inadequação na utilização dos métodos contraceptivos, além da falta de serviços assistências, onde possam buscar orientações e atendimento.

Tal realidade alerta para a necessidade de maior envolvimento de profissionais da saúde, da educação, família e comunidade, garantindo qualidade de vida aos adolescentes.

Palavras-chave Adolescente, Gravidez, Contracepção 


\section{Introdução}

A anticoncepção é um tema muito importante, especialmente na adolescência, considerando a relevância social conferida pela ocorrência de gravidez nessa faixa etária e pela possibilidade de exposição às doenças sexualmente transmissíveis (DST) e AIDS.

O conhecimento sobre os métodos contraceptivos e os riscos advindos de relações sexuais desprotegidas são fundamentais para que os adolescentes possam vivenciar o sexo de maneira adequada e saudável, assegurando a prevenção da gravidez indesejada e das DST/AIDS, além de ser um direito que possibilita cada vez mais, ao ser humano, o exercício da sexualidade desvinculado da procriação.

Definida como o período etário compreendido entre 10 e 19 anos completos, 1 a adolescência é uma fase do desenvolvimento que marca a passagem da infância à vida adulta, caracterizada por transformações biopsicossociais, determinadas por fatores genéticos e ambientais. 2 Marcada também por especificidades emocionais e comportamentais que se refletem na saúde sexual e reprodutiva, torna os adolescentes mais vulneráveis aos mesmos riscos aos quais muitos adultos estão expostos.

A cada ano, registra-se o nascimento de mais de 14 milhões de crianças, pertencendo suas mães ao segmento adolescente. $\mathrm{O}$ índice de jovens que têm seu primeiro filho em torno de 18 anos varia de $1 \%$ no Japão a $53 \%$ na Nigéria. 3

Segundo Cavasin e Arruda, 4 no Brasil o parto representou a primeira causa de internação de adolescentes do sexo feminino no Sistema Único de Saúde. Na faixa etária de 15 a 19 anos, o principal motivo de internação das mulheres foi à gravidez, o parto e o pós-parto, e as complicações decorrentes desses eventos. Em todas as regiões do país $80,3 \%$ das internações nessa faixa etária são decorrentes desses motivos: Norte $-79,5 \%$; Nordeste- $81,1 \%$; Sudeste- 80,9\%; Sul 77,6\% e Centro-Oeste- 80,2\%. A Pesquisa Nacional sobre Demografia e Saúde realizada pela Sociedade Civil do Bem Estar Familiar no Brasil (BEMFAM) 5 em 1996, revelou que $18 \%$ das adolescentes já tiveram pelo menos um filho, sendo esta taxa mais elevada nas áreas rurais (24\%) do que nas áreas urbanas (17\%).

No município de Bauru, estado de São Paulo, estudos recentes revelaram que as adolescentes foram responsáveis por aproximadamente $20 \%$ a $25 \%$ das gestações. ${ }^{6,7}$ Nesse contexto, o aumento na incidência de gravidez tem sido identificado como um problema de magnitude no cenário da saúde pública.
Os motivos pelos quais as adolescentes engravidam são diversos destacando-se a falta de informação, fatores sociais, falta de acesso a serviços específicos para atender essa faixa etária, o início cada vez mais precoce de experiências sexuais e a insegurança do adolescente em utilizar métodos contraceptivos. ${ }^{8}$

Berquó 9 assinala que a precocidade é maior para os jovens atualmente, e verificou uma média para o início da atividade sexual entre 14,5 anos para os homens e de 15,2 para as mulheres em uma população com faixa etária de 16 a 19 anos. Para a mesma variável em uma população acima de quarenta anos, a autora constatou média de idade para homens de 18,4 anos e para mulheres de 20,6 anos.

Em trabalho que buscou avaliar a saúde reprodutiva de adolescentes do município de Botucatu, São Paulo, fazendo parte de um projeto multicêntrico em Políticas de Saúde Pública, realizado por meio do emprego de questionários no ambiente domiciliar, Silva et al. 10 constataram que de um total de 421 adolescentes, 301 (71,5\%) referiram já ter "ficado" pelo menos uma vez. A média de idade entre os adolescentes foi de 12,1 anos, enquanto que entre as adolescentes foi de 12,8 anos. Ademais 115 (27,3\%) já haviam tido relações sexuais, ocorrendo, em média, entre eles aos 15,0 anos e entre elas aos 15,2 anos.

Além do início da atividade sexual precoce, os jovens, na maioria das vezes, iniciam a vida sexual sem proteger-se, expondo-se a gravidez indesejada e não planejada. Estudos realizados na América Latina têm mostrado que menos de $20 \%$ dos homens e de $15 \%$ das mulheres usam algum método anticoncepcional na primeira relação sexual.11

Raramente, na prática clínica ou no contato com jovens no ambiente escolar, depara-se com um adolescente que negue ter recebido informações sobre opções contraceptivas, porém vários estudos revelam o uso inadequado, assim como relações sexuais desprotegidas e deficiência dos serviços de saúde para atendimento e acompanhamento de jovens nessa faixa etária.

Segundo Guimarães et al.,12 a escola não tem representado para os adolescentes fonte de informação expressiva sobre anticoncepcionais. Quanto à importância de conhecimento sobre sexualidade, Moreira13 destaca a necessidade de criação de programas de atualização, informação para profissionais e pacientes ressaltando que, para escolares o processo deve ser iniciado já no ensino fundamental.

Partindo-se do pressuposto que o despertar e o exercício da sexualidade implicam na possibilidade da utilização de serviços que prestam assistência 
com qualidade, o desafio atual é proporcionar aos adolescentes assistência integral garantindo-lhes privacidade, confidencialidade e apoio sem juízo de valor. ${ }^{14-16}$

A inadequação de métodos contraceptivos por parte dos adolescentes, decorrente de fatores socioeconômicos e culturais, trás conseqüências danosas não só para essa faixa etária, como também para toda a sociedade. Baseando-se nos estudos da literatura e na prática clínica, os quais nos alertam para tal realidade, o objetivo deste artigo é contribuir com informações científicas, para profissionais da saúde e educação, envolvidos com essa faixa etária.

\section{Tipos de metodos contraceptivos}

Os diferentes métodos contraceptivos são conhecidos pelos profissionais da saúde, da educação e também pela maioria da população em idade reprodutiva. Porém, a eficácia e a utilização correta nem sempre é explorada, principalmente com os adolescentes, os quais têm iniciado cada vez mais precocemente as atividades sexuais sem, contudo receberem ou buscarem informações acerca da contracepção.

Segundo o Ministério da Saúde, 14,15 a assistência em anticoncepção pressupõe oferta de todas as alternativas de métodos contraceptivos, assim como o acompanhamento clínico-ginecológico da adolescente referente ao método elegido. 17 Tais métodos se dividem de acordo com o mecanismo de ação: a) métodos comportamentais que estão embasados na auto-observação que ocorre no organismo ao longo do ciclo menstrual, sendo necessário que as usuárias tenham ciclos menstruais regulares e que exista cumplicidade entre o casal (Ogino-Knaus, temperatura basal corporal, muco-cervical ou Billings); b) os métodos de barreira que consistem em obstáculos mecânicos ou químicos à penetração dos espermatozóides no canal cervical (preservativo: masculino e feminino, diafragma, geléias espermicidas); c) métodos hormonais (oral-pílula, injetáveis e implante) cuja finalidade básica é impedir a concepção; d) dispositivo intra-uterino (DIU), que atuam impedindo a fecundação; e) métodos cirúrgicos ou esterilização (ligadura das trompas e a vasectomia); f) contracepção de emergência, método alternativo hormonal oral que evita a gravidez quando ingerido até 72 horas após a relação sexual desprotegida. ${ }^{14,15,18-22}$

Duarte et $a l .23$ afirmam que a escolha do método contraceptivo deve ser responsabilidade do casal. Nesse sentido os autores ressaltam a importância da assistência integral a saúde sexual e reprodutiva de homens e mulheres, com implantação de programas que incluam a reflexão acerca da dinâmica das relações e dos papéis sociais.

\section{Conhecimento e comportamento contracptivo}

Diversos estudos buscam verificar o conhecimento e o comportamento contraceptivo em adolescentes com objetivo de analisar tal realidade e propor estratégias que promovam a saúde sexual e reprodutiva para esse grupo etário especificamente.

A sexualidade na adolescência é importante, e os profissionais da saúde devem estar preparados para respeitar a autonomia de livre escolha e ofere-cer informações e acompanhamento adequado, lhes garantido assistência de qualidade. Destaca-se também que a idade não deve constituir restrição ao uso de qualquer método anticoncepcional na adolescência depois da menarca. 24

A menarca mais precoce vem expondo a adolescente aos riscos de uma gravidez em idades também precoce e vários estudos referem que a média de idade da menarca no Brasil está em torno de 12 a 13 anos de idade. 25,26

Dentre os motivos mencionados pelas adolescentes sobre a falta do uso da anticoncepção, encontra-se a dificuldade de diálogo com o parceiro, a qualidade e/ou inadequação da informação a respeito da contracepção e reprodução, assim como sobre o uso correto dos métodos anticoncepcionais. 27

Uma pesquisa em seis escolas de diferentes níveis socioeconômicos, com 128 estudantes de ambos os sexos, entre 11 e 19 anos selecionados ao acaso, revelou que $81,7 \%$ conheciam alguns métodos anticoncepcionais, sendo o preservativo e a pílula os mais citados. Apesar do conhecimento a maioria referiu não utilizá-los por não valorizar as chances de gravidez ou por mero esquecimento. 28

Outro dado relevante se relaciona ao abandono do método contraceptivo pelo adolescente, Davim ${ }^{29}$ realizou um estudo com 36 adolescentes na faixa etária entre 13 a 19 anos de idade buscando identificar, além dos métodos contraceptivos conhecidos e utilizados, as causas que as levaram a abandonar a utilização dos referidos métodos. Os resultados indicaram desconhecimento acerca da contracepção, da gravidez, dos riscos de uma gestação precoce, destacando-se que a grande maioria abandonou os métodos de contracepção pelo desejo de engravidar.

Lescano 30 investigando 173 adolescentes na Maternidade do Hospital de Apoio de Sullana, no 
Peru constatou que $91,9 \%$ delas revelaram algum conhecimento das opções anticonceptivas existentes, sendo a pílula, a contracepção hormonal injetável, o DIU e o preservativo masculino os métodos mais citados. Destaca ainda que as principais fontes de informações foram obtidas por meio de profissionais da saúde, da escola, parentes e vizinhos.

A responsabilidade sexual frente à anticoncepção em adolescentes com idades variando entre 13 e 18 anos foi motivo de um estudo realizado por Peláz Mendonça et al. 31 o qual revelou que apesar de $82 \%$ dos jovens apresentarem conhecimento sobre os métodos contraceptivos, apenas $8 \%$ referiram ter se protegido na primeira experiência sexual. Quanto ao método mais conhecido, foi o preservativo masculino o mais citado, sendo que a principal razão apontada para a não utilização de tal método está vinculada à diminuição das sensações durante a relação sexual.

A imprevisibilidade da relação sexual também foi citada pelos adolescentes como motivo do não uso de contraceptivos, conforme evidenciaram, Ferreira et al.32 em um grupo de 36 adolescentes do sexo feminino com idades entre 11 e 17 anos. Dentre os métodos contraceptivos mais utilizados destacouse o preservativo masculino $(66,7 \%)$ seguido pela pílula $(36,7 \%)$.

Culturalmente a prevenção da gravidez tem sido responsabilidade da mulher. Almeida et al.33 comparando o conhecimento e a utilização de métodos contraceptivos em ambos os sexos, constataram em uma amostra de 4774 escolares matriculados em 12 escolas da Rede Estadual Pública no Estado da Bahia, diferença estatisticamente significante já no decorrer da adolescência, para o sexo feminino. Quanto à prevalência do uso de métodos anticoncepcionais na primeira e por ocasião da última relação sexual, o estudo registrou $36,6 \%$ para o sexo masculino e $46,4 \%$ para o sexo feminino.

Em contrapartida, Duarte et al. 23 investigando a participação masculina no uso de métodos contraceptivos em uma população de 910 homens adultos, destacaram em seus achados um maior compromisso masculino referente à contracepção. Quando comparados aos dados populacionais existentes, tais informações confirmaram uma tendência nacional observada na década de noventa. Segundo os autores uma comparação realizada por meio dos dados da Pesquisa Nacional sobre Demografia e Saúde (PNDS) entre os anos de 1986 e 1996 revelou uma proporção três vezes maior de usuários de vasectomia e duas vezes maior em usuários de preservativo. Para os autores os resultados do estudo possivelmente foram influ- enciados pelo elevado perfil de escolaridade da amostra investigada. Tais dados podem não corresponder à realidade do grupo dos adolescentes, os quais tem iniciado cada vez mais precocemente suas experiências sexuais. 9

Neste contexto, Belo e Silva ${ }^{34}$ pesquisando o conhecimento, atitudes e práticas em relação ao uso de métodos contraceptivos evidenciaram que o nível de escolaridade no grupo dos adolescentes não modificou o conhecimento ou influenciou na contracepção conforme estudo realizado com a participação de 156 gestantes entrevistadas no decorrer da primeira consulta do pré-natal realizadas no Centro de Atenção Integral a Saúde da Mulher (CAISM). Os autores consideram que tal aspecto aponta para as dificuldades ou até mesmo para a inexistência de diálogos familiares que favoreceriam avanços significativos na apropriação de conhecimentos refe-rentes à prevenção da gravidez não planejada.

Lima et al. 35 por meio de uma análise qualitativa buscaram conhecer as percepções e práticas de adolescentes grávidas e de seus familiares em relação à gravidez. A casuística foi composta de 19 gestantes que realizaram o pré-natal nas unidades de saúde da Ilha do Chié, pertencente ao Distrito Sanitário II, da cidade do Recife, e 14 pessoas responsáveis pelos cuidados dessas adolescentes. Constataram a necessidade de aprender presente no discurso das adolescentes, verificando que $68,4 \%$ consideravam importante o conhecimento sobre métodos contraceptivos e a prevenção da gravidez. Dentre os meios mais citados encontraram: realização de palestras $(46,1 \%)$, orientações sobre sexualidade e prevenção da gravidez $(23,0 \%)$, disponibilização de métodos anticoncepcionais orais $(23,0 \%)$ e injetáveis $(15,3 \%)$, e preservativos masculinos $(15,3 \%)$. Citaram ainda que para a concretização de tais objetivos deve-se buscar estratégias de promoção que estejam mais próximas das necessidades geradas no contexto sóciocultural em que esse grupo populacional se vincula.

\section{Programas assistenciais}

O panorama da contracepção na adolescência deixa clara a necessidade não só de ampliar o acesso a serviços especializados, como também a importância de contextualizar no processo educativo, o conhecimento referente à sexualidade, destacando-se a promoção da saúde e a inclusão da família e da comunidade.

De acordo com Osis et al.36 no Brasil à partir de 
1984 com a implantação do CAISM, buscou-se a qualidade referente ao atendimento na área do planejamento familiar, o que levou alguns serviços públicos de saúde a implantarem metas educativas as mulheres sobre contracepção. Os autores apontam ainda que as estratégias neste âmbito de ação devem contemplar um projeto abrangente envolvendo a sociedade, não centralizando apenas nos serviços de saúde. Neste sentido, as ações de planejamento familiar devem ser divulgadas por meio de diálogos em escolas, centros comunitários, unidades de saúde e reuniões com diferentes grupos etários. Preconizase a realização de materiais de divulgação adequada a cada grupo específico, sugerindo também a importância da utilização dos meios de comunicação em massa. 15

Em 1989, foi implantado o Programa de Saúde do Adolescente (PROSAD), para ambos os sexos, no grupo etário de dez a 19 anos de idade, tendo como enfoque prioritário à atenção à sexualidade e a saúde reprodutiva. 14 Contudo, os dados referentes à gra-videz indesejada e não planejada na adolescência vêm demons-trando índices alarmantes, apresentando taxas de $30 \%$ a $50 \%$ de mulheres que tiveram um parto antes dos vinte anos, as quais declararam a gravidez como não desejada, confirmando a necessidade de oferecer atendimento e acompanhamento integral, antes mesmo do início da vida sexual. 11,35-36

\section{Referências}

1. OMS (Organização Mundial da Saúde). El embarazo y el aborto en la adolescência. Ginebra; 1975. (Informe Técnico, n. 583)

2. Goldberg TBL, Jaehn SM, Camapana AP, Kfouri JRN, Simões ACP, Curi PR. Avaliação do desenvolvimento afetivo-social do adolescente na faixa etária dos 15 aos 18 anos: estudo com adolescentes do município de Botucatu, São Paulo: características sociais e interação familiar. J Pediatr (Rio J) 1987; 63: 213-7.

3. Alan Guttmacher Institute. Into a new world. Young women sexual and reproductive lives. New York; 1998.

4. Cavasin S, Arruda S. Educação sexual e comunicação para adolescentes. In: Vieira EM, Fernandes MEL, Bailey P, Mckay A, organizadores. Seminário gravidez na adolescência; 1998 jul 30-31; Rio de Janeiro, RJ. Rio de Janeiro: Associação Saúde da Família; 1998. p. 110-8.

5. BEMFAM (Sociedade Civil do Bem Estar Familiar no Brasil). Pesquisa Nacional de Demografia e Saúde 1996. Rio de Janeiro; 1997.

6. Soares EO. Estudo epidemiológico descritivo de recémnascidos vivos de mães adolescentes e adultos, no município de Bauru (SP), 1998 [dissertação mestrado]. Botucatu: Faculdade de Medicina da Universidade Estadual Paulista; 2001.

\section{Comentários finais}

Os estudos sobre a adolescência e sexualidade evidenciam a necessidade de abordagem clara e livre de preconceitos, envolvendo família, escola, comunidades religiosas, ambientes prestadores de assistência à saúde e de formação profissional habilitada e capacitada. Faz-se necessária a implementação de estratégias que permitam aos jovens desse grupo etário conscientizar-se sobre a importância que envolve a saúde sexual e reprodutiva e dialogar, sem juízo de valor, sobre suas dúvidas e vivências, o que poderia prevenir e garantir uma adolescência saudável.

Fica pois, evidente a responsabilidade de toda sociedade no que tange a promoção e a qualidade de vida do adolescente.

"O uso de contraceptivos mostra uma atitude positiva frente a sexualidade, mas também um grau de maturidade e autoestima próprios de quem projeta o futuro negociando com o presente as suas decisões". (Ramos; 2001: 31). 37

\section{Agradecimentos}

As autoras agradecem à Ilana Goldberg pela versão do resumo para o inglês.

7. Lombardi HF. Oportunidades de diagnóstico precoce do HIV em gestantes do município de Bauru [dissertação mestrado]. Botucatu: Faculdade de Medicina da Universidade Estadual Paulista; 2002.

8. Kraft P. Sexual knowledge among Norwegian adolescents. J Adolesc 1993; 16: 3-21.

9. Berquó E, coordenadora. Comportamento sexual da população brasileira e percepções do HIV/AIDS. Brasília (DF): Ministério da Saúde; 2000. (CEBRAP: Série Avaliação,n.4)

10. Silva RC, Almeida MAS, Iyda M, Carandina L, Goldberg, TBL. Saúde reprodutiva entre adolescentes na cidade de Botucatu. Cienc Saúde Coletiva 2003; 8: 757 [Abstract].

11. Diaz J, Diaz M. Contracepção na adolescência. Cad Juv Saúde Desenvol 1999; 1 : 249-57.

12. Guimarães AMDN, Vieira MJ, Palmeira AJ. Informações dos adolescentes sobre métodos anticoncepcionais. Rev Latino Am Enferm 2003; 11: 293-8.

13. Moreira LMV. Perda fetal espontânea: avaliação do nível de conhecimento e influência dos fatores de risco [dissertação mestrado]. Bauru: Faculdade de Odontologia da Universidade do Sagrado Coração; 2001.

14. Ministério da Saúde. Secretaria de Políticas de Saúde. Área Técnica de Saúde da Mulher. Assistência em planejamento ramiliar: manual técnico. 4. ed. Brasília (DF); 2002. 
15. Ministério da Saúde. Planejamento familiar: manual para o gestor. Brasília (DF); 2002.

16. Saito MI, Leal MM. Fórum 2002: Contracepção, adolescência e ética. [on line].Disponível em: http:// www.nutrociencia.com.br/upload/planejamento $\% 20$ familiar.pdf. [2004 fev 13].

17. Saito MI, Leal MM. Aspectos éticos da contracepção na adolescência. Rev Assoc Med Bras 2003; 49: 234-4.

18. Vilela ALM. Métodos anticoncepcionais. Anatomia e fisiologia humana. Disponível em: http.www.afh.bio.br/ básicos/repro8.htm. [2004 fev 6].

19. Aleixo Neto A. Contracepção na adolescência. Disponível em http://www.medicina.ufmg.br/edump/gob/dumpgo2.htm [2003 out 20].

20. FEBRASGO (Federação Brasileira das Associações de Ginecologia e Obstetrícia). Saúde do adolescente: manual de orientação. Disponível em: www.febrasgo.org.br./ manuais.htm. [2004 fev 6].

21. Corleta EE. Anticoncepção: métodos hormonais. ABC da Saúde, 2001 Disponível em: www.abcdasaude.com.br/ artigo.phd. [2004 fev 9].

22. Hardy E, Duarte GA, Osis MJD, Arce XE, Possan M. Anticoncepção de emergência no Brasil: facilitadores e barreiras. Cad Saúde Pública 2001; 17: 1031-5.

23. Duarte GA, Alvarenga AT, Osis MJD, Faúndes A, Sousa MH. Participação masculina no uso de métodos contraceptivos. Cad Saúde Pública 2003; 19: 207-16.

24. WHO (World Health Organization). Improving access to quality care in family planning. Medical eligibility criteria for contraceptive use. Geneva; 1996.

25. Colli AS. Crescimento e desenvolvimento físico. In: São Paulo [Estado]. Secretaria de Saúde. Comissão de Saúde do Adolescente. Adolescência e saúde. São Paulo: Paris Ed.; 1998. p. 43-58.

26. Almeida MAS. Treze meninas e suas histórias: um estudo sobre mães adolescentes [tese doutorado]. Araraquara: Faculdades de Ciências e Letras da Universidade Estadual Paulista Júlio de Mesquita Filho; 2001.

27. Romero MI, Maddaleno M, Silber TJ, Munist M. Salud reproductiva. In: Silber TJ, Munist MM, Maddaleno M, Ojeda ENS, organizadores. Manual de medicina de la adolescencia. Washington (DC); 1991. p. 473-82.

Recebido em 24 de março de 2004

Versão final apresentada em 5 de setembro de 2005

Aprovado em 26 de novembro de 2005
28. Bruno ZV, Sousa MA, Teixeira LGM, Silva RB, Silva RB, Guanabara EM, Oliveira FC. Sexualidade e anticoncepção na adolescência: conhecimento e atitude. Reprod Clin 1997; 12: $137-40$.

29. Davim RMB. A prática da contracepção: causas de abandono na utilização de métodos contraceptivos por adolescentes [dissertação mestrado]. João Pessoa: Centro de Ciências da Saúde, Universidade da Paraíba; 1998.

30. Lescano EL. Adolescência e anticoncepção: um estudo de adolescentes atendidas na Maternidade do Hospital de Apoio, Sullana, Peru [dissertação mestrado]. São Paulo: Faculdade de Saúde Pública da Universidade de São Paulo; 2001.

31. Pelaz Mendonza J, Rodrigues Pons O, Bermúdez Sánchez R. Adolescente varon y anticoncepcion. Rev Cubana Obstet Ginecol 1998; 24: 5-12.

32. Ferreira MLSM, Galvão MTG, Costa ES. Sexualidade da adolescente: anticoncepção. Rev Bras Med 2000; 57: 8-15.

33. Almeida MCC, Aquino EML, Lynne G, Magnani RJ. Uso de contracepção por adolescentes de escolas públicas na Bahia. Rev Saúde Pública 2003; 37: 566-75.

34. Belo MAV, Silva JLP. Conhecimento, atitude e prática sobre métodos anticoncepcionais entre adolescentes gestantes. Rev Saúde Pública 2004; 38: 479-87.

35. Lima CTB, Feliciano KVO, Carvalho MFS, Souza APP, Menabó JBC, Ramos LS, Cassundé LF, Kovacs MH. Percepções e práticas de adolescentes grávidas e de familiares em relação à gestação. Rev Bras Saúde Matern Infant 2004; 4: 71-83.

36. Osis MJD, Duarte GA, Crespo ER, Espejo X, Pádua KS. Escolha de métodos contraceptivos entre usuárias de um serviço público de saúde. Cad Saúde Pública 2004; 20: 1586-94.

37. Ramos R. Dificuldade no acesso à contracepção. Sex Plan Fam 2001; 29: 29-31. 